in the small of the back. Pulse 12 I and full; respiration 20. She suffered from dyspncea on going up stairs rapidly. I referred her back to Dr. B. for further examination. He found both albumen and casts in urine. Dr. B. put her on treatment, milk diet, etc. In five weeks the fundus had improved wonderfully, and vision increased to $20 / 40$, or half of perfect. Miss B. is a very well-developed girl, and had had no sickness recently.

In all of these cases the first symptom was the loss of vision, with no other symptom that would have attracted the attention of the physician. Many cases have been reported in which the eye symptoms preceded for some weeks the albumen and casts. Of course in these cases the ophthalmoscope is invaluable. Such cases would indicate that the disease is improperly named. They appear also to support Sir William Gull's theory, that Bright's disease originates as a disease of the arterioles, not only of the kidneys, but of the whole circulatory system. There is a case on record in which the left eye alone, of a patient 43 years old, was affected with all the subjective and objective symptoms of parenchymatous nephritis. At the autopsy, the right kidney was absent, its place being occupied by a hypertrophied lobe of the liver; there was nothing to indicate that there had ever been a right kidney except the presence of a supra-renal capsule. The left kidney (remember it was the left eye that was diseased) was in its normal position; was considerably hypertrophied, and had the characteristics of the large white kidney of parenchymatous nephritis. Cases of unilateral retinitis albumenurica are not very common. Case 5 is the first I ever saw. The right eye of this patient is, and has been all along, perfect. Schweigger says: "It always affects both eyes, although not always in the same degree." The changes observed in retinitis albumenurica are considered by Liebreich as so constant in this form of inflammation, that Bright's disease may be diagnosticated with certainty by the ophthalmoscope.

Sometimes in what is known as descending neuritis, the symptoms very closely resemble those of nephritic retinitis. What are the changes seen in nephritic retinitis? Besides the usual symptoms of neuro-retinitis, such as hyperæmia of nerve and retina, we find grouped around the macula lutea, white spots arranged in a stellate form, and looking very much as if some one had splattered white paint on the surface with a brush; this is in the earlier stages. Later the spots unite in a large, white glistening spot, surrounding the optic nerve, often not extending entirely to it, and reaching as far as the equator. Dipping in and out of this white substance, can be seen the blood-vessels, which have here and there broken down, resulting in hæmorrhages into theretina.

The three symptoms most characteristic of nephritic retinitis are: the bright stellate spots around the macula, the white mound surrounding the nerve, and the spots of hæmorrhage. The choroid and vitreous are often involved. Vision varies greatly. One of the worse cases I ever saw (Case I) reported, had perfect vision. Vision is very rarely reduced to zero. Schweigger says: "This form of retinitis may be developed in all cases in which albumenuria has existed for some time: This is most frequently the case in chronic Bright's disease; still the existence of retinal disease has also been demonstrated in connection with crompous nephritis, with amyloid degeneration of the kidneys, and in the passive hyperamia of the kidneys in pregnancy."

Retinal disease occurs in about seven per cent. of all cases of Bright's disease. Occurring most frequently in the most insidious form of Bright's disease (the cirrhotic), it makes the ophthalmoscopic examination, therefore, the more important.

Let us for a moment glance at the pathology of the changes observed in retinitis Brightii. The hæmorrhages are the result of fatty degeneration of the coats of the vessels. The striated appearance of the infiltration into the retina and optic nerve is the result of the formation of the connective tissue that support the nerve fibers. The large, white surface surrounding the optic nerve, and the stellate spots around the macula, depend upon fatty degeneration of the cellular and connective tissue elements of the retina, more especially of the external granular layer, with exudation into the retina. The stellate appearance at the macula, is due to the peculiar anatomical arrangement of the radial connective tissue fibres at the yellow spot. The optic nerve fibres also often undergo sclerosis, and are seen in groups of small glistening spots, which are of great importance, especially as to prognosis: They closely resemble the spots of connective tissue fatty degeneration, but are differently located, being more superficial, or in front of the blood vessels, the latter being in the external granular layer, or back of the blood vessels. Often changes occur in the nerve elements of the internal layers. 'The cheroidal changes observed are round masses of sclerosed substance, corresponding to the degenerated portions of the retina. (Virchow). A peculiar embolism of the fine ciliary arteries has been noticed. Proliferation of the connective tissue of the adventitial coat of the retinal vessels often occurs, as seen by bright stripes accompanying the vessels.

The connecting link between the disease of the kidney and that of the retina has not been satisfactorily demonstrated. Some have supposed it to be due to impairment of nutrition, others to the urea, and others again to the albumen. There are many cases on record that upset all these theories.

The best prognosis is afforded in these cases, following the exanthemeta, typhoid fever and the advanced stages of pregnancy. No special treatment to the eye is indicated beyond rest and avoidance of bright lights.

\section{CONCUSSION OF THE BRAIN, FOLLOWED BY FATAL MENINGITIS.}

BY H. MOULTON, M.D.,

$$
\text { OF STVART, IOWA. }
$$

On the afternoon of June I3, two boys, aged each about fifteen years, were fighting each other in rather close quarters, when one drew from his pocket a heavy knife, closed, and with a short, quick, upward 
stroke, he inflicted in the left temporal region of the other a wound, from which blood poured freely. The bystanders, thinking the blow too light to have produced a serious injury, referred the hæmorrhage to the nose. When I reached the spot, a few minutes later, blood still flowed, encouraged by the injudicious local use of water.

Hamorrhage being checked by pressure, the boy fainted. Soon reviving, he was brought to my office, where the wound was dressed. The sharp corner of the knife handle had made, not a deep cut, in the mass of temporal muscle, about one inch above the mid point of a line drawn from the external canthus of the eye to the external auditory meatus. There was no sign of fracture, nor symptom of concussion or compression; and there was no paralysis. The pupils of the eyes were slightly dilated, otherwise normal. The boy did not lose consciousness, nor fall or even stagger after the blow. He was pale and weak from the loss of blood, complained of slight frontal headache, and vomited once before leaving the office. At 6 P.M., one hour after the accident, he walked with some assistance to a carriage, and was taken home. At this time his temperature was normal, and pulse weak and moderately accelerated. During the evening he vomited, had a chill, lasting a half an hour or so, became feverish, and passed a restless night, with some delirium.

On seeing him next day, at 9:30 A.M., the temperature in the axilla was $104{ }^{\circ} \mathrm{F}$., respiration 28 , and the pulse not very vigorous at 120 per minute. The head ached, the skin was dry, the eyes normal in appearance, and the special senses not perverted. The patient was restless in bed, but complied intelligently with all my requests and made known all his wants. Owing to the recent free bleeding, depletory measures were not adopted, but cold to the head and sponging of the body were ordered, and a single $5 \mathrm{gr}$. dose of quinine administered.

I :30 P.M.-Temp. I04 F.; resp. 30 ; pulse $\mathbf{2} 20$. Patient was more delirious, and had a mild general convulsion.

4 P.M.-Temp. $104.2^{\circ}$; resp. 32 ; pulse 13 o. Conjunctivie were now congested, and the pupils of the eyes contracted, but sensitive to light. Delirium continued, and there had been occasional spasm of some of the muscles of the face and forearm. Large doses of bromide of potassium had been given, and these symptoms soon subsided. Six grains of calomel were administered at this time.

6:30 P.M.-Temp. I0 $5^{\circ}$ F.; pulse I 30 ; resp. rapid. Patient was put into the wet pack, and at 8 o'clock had a temperature of $103.5^{\circ} \mathrm{F}$. He was now rapidly becoming unconscious, and soon was in a state of complete stupor.

During the night, under the use of cold and the ice cap, the temperature ranged from $103^{\circ}$ to $104^{\circ}$ F. The pulse became more feeble, and respiration rapid and shallow. There was some tonic contraction of the flexors of the forearm. The freces and urine were passed involuntarily into the bed.

June 15,7 A.M. - Temp). I0 $4.5^{\circ}$ F.; resp. 40 ; pulse I38. Hands and feet cold, skin moist, and the pupils of the eyes irresponsive to light.
Io A.M.--Cyanosis developed, with the pulse imperceptible at the wrist.

At I 2 M., 43 hours after the receipt of the injury, death took place from apncea.

The plan of treatment followed was, to reduce temperature by sponging the arms, neck and trunk, and by the wet pack; to reduce temperature and lessen local inflammation by cold to the head, using first simple cold water, afterwards an ice cap; to lessen intra-crannial congestion and quiet nervous excitement by the use of large doses of bromide of potassium; and to strengthen the heart by the administration of digitalis begun early. Consultation was had during the progress of the case, with Dr. J. H. Kersey.

At the autopsy, an account of a former unfortunate circumstance occurring in the practice of another, we were enjoined not to remove the calvarium and limited to an examination at the seat of injury. The wound was laid open and found to penetrate about half way to the skull, through the muscles and fascia. After dissecting up a flap from before backwards, the skull presented no mark of violence. The trephine, applied over the anterior inferior angle of the parietal bone, exposed the dura mater and the branch of the middle meningeal artery uninjured. There was no extravasation between the dura and the bone. A finger passed in at the opening, and swept around as far as it could reach, detected no sign of fracture of the internal table of the skull. The dura was but slightly congested. Underneath it were a number of small blood clots which were removed. Others were brought into sight by pressing aside the brain substance, but no large ones appeared. The pia mater and superficial brain substance were intensely red and congested and tumid.

In the light of this limited examination, the conclusion was that the vessels of the pia mater were ruptured by, and a general meningitis followed the concussion, and that this result was favored by great intra-cranial congestion, due to excitement and the heat of the day. There may have been other injury to the brain which we could not see. Mr. Holmes would place this among "cases not classifiable," in which the usual symptoms of concussion are absent, and hæmorrhage is not sufficient to produce compression, but in which a fatal injury is done, and "some laceration of the brain or extravasation into the membranes appears to have been always found."

Stuart, Iowa, July 8 th, 1885 .

\section{MEDICAL PROGRESS.}

\section{ANATOMYZAND PHYSIOLOGY.}

The Eifectrical Excitability of the Brain.This is a subject on which M. Vulpian made a report to the Académie des Sciences, on March 23. The doctrine of the cortical motor localisations depends on two kinds of experimental proof; one being the effects of destruction of cortical regions, the other the motor effects of electrisation of these regions. The latter, the only one which Vulpian 